\title{
Transporte urbano sostenible: medidas desde la administración y transporte público como alternativa en Bogotá D.C.*
}

\author{
Sustainable urban transport: administration and \\ public transport measures as an alternative in \\ Bogota, D. C.
}

Sonia Jiménez Murillo Munar**

\section{Resumen}

En medio de los problemas ambientales generados por el transporte urbano, y la preocupación mundial por la sostenibilidad ambiental, surgen en Bogotá, D.C., como en muchas otras ciudades, medidas para contrar restar los efectos contaminantes de las fuentes móviles, sobre todo en la calidad del aire. En este artículo se comentan brevemente las medidas implementadas en Bogotá, D.C. para desincentivar el uso del automóvil privado y crear conciencia para generalizar el uso de modos alternativos de transporte; además, se analiza la condición del transporte público en la ciudad, como opción en la búsqueda de una movilidad sostenible. Se presenta un indicador de accesibilidad al transporte público, que combina diferentes variables para determinar el cubrimiento del sistema y concluir si es posible acoger a todos los usuarios de la ciudad, de manera satisfactoria.

Palabras claves: Bogotá, D. C.; M ovilidad, Transporte público urbano.

Este artículo presenta al gunos aspectos desarrollados durante la investigación y el aboración de la tesis de Maestría en Geografía: "En busca de la inserción de nuevos modos de transporte - no tradicionales- en I a movilidad de Bogotá: Una alternativa de accesibilidad a escal a local".

* Uni versidad Nacional de Colombi a. E-mail: sjmurillom@nal. edu.co 


\section{Abstract}

In the midst of environmental problems generated by urban transportation, and a global concern for environmental sustainability, measures have been taken in Bogota, as in many other cities, in order to mitigate environmental pollution of the air particularly from mobile sources. In this paper instruments implemented in Bogotá, D. C. to discourage the use of private automobiles as a means of transportation and create consciousness on the generalization of alternatives means of transportation are briefly discussed. Furthermore, the condition of public transportation in the city is analyzed, as an option of sustainable mobility. An indicator of public transportation accessibility is presented. This indicator gathers different variables in order to determine the coverage of the system and determine if it is possible to accommodate all public transportation users of the city in a satisfactory manner.

Key words: Accessibility, Mobility, Public urban transportation. 


\section{Introducción}

La investigación base, de la cual parte este artículo, reconoce la relación entre transporte y estructura urbana, y aborda los principales problemas geográficoambientales, relacionados con el transporte urbano y una preocupación mundial por la sostenibilidad ambiental, que hoy busca soluciones en el uso de transportes alternativos para tratar de minimizar el impacto contaminante -al aire y el ambiente por ruido- del uso del automóvil masivamente en la ciudad, así como reducir distancias y tiempos de viajes obligatorios, mediante una nueva estructuración urbana.

La investigación exploró otra alternativa para el mejoramiento del transporte y la movilidad humana en la ciudad, para mejorar la accesibilidad de todos los individuos, sin aumentar la contaminación y problemas ambientales, hacia una mayor interacción y articulación del espacio urbano, con el reconocimiento de las diferencias físicas naturales y construidas de los espacios, como las características socioeconómicas de los usuarios y las técnicas de los modos de transporte.

Así, se estudió la ciudad de Bogotá, D.C. y en ella las dificultades de movilidad de sus habitantes, por congestión, falta de acceso al transporte público, largas distancias y tiempos de viaje (Duarte, 2004), derivados de la organización del transporte en la ciudad y la falta de articulación con el modelo de estructura urbana que se propone desde la planificación (Alcaldía Mayor de Bogotá, 2004; Alcaldía Mayor de Bogotá y STT 2004; Alcaldía Mayor de Bogotá y STT 2005a; Alcaldía Mayor de Bogotá y STT, 2006b; Alcaldía Mayor de Bogotá y STT, 2006c;). Pero, además, como una consecuencia de la política para abordar el ordenamiento y las acciones sobre el territorio urbano desde una mirada general, que omite las escalas y las necesidades locales, genera desigualdades de accesibilidad al transporte público y refuerza las dificultades de acceso a los servicios urbanos, desde algunas comunidades.

Se propone, como aporte para mejorar la movilidad y la accesibilidad, el comprender que la ciudad no es homogénea, sino que posee áreas diferenciadas por sus características físicas, socio-culturales y económicas, e instaurar modos de transporte alternativos a los actuales, que reconozcan esa realidad para prestar un servicio de manera mas eficiente y acorde con sus capacidades y requerimientos técnicos, articulándose con el sistema de movilidad general.

Este artículo retoma esas reflexiones y revisa, en Bogotá, D.C., los problemas de movilidad y las medidas que, desde la administración, buscan desincentivar el uso del automóvil; plantea el transporte público como solución para acoger a la mayoría de los bogotanos, incluyendo a quienes se bajen de su carro propio, centrándose en el análisis de cubrimiento de éste, para evaluar la coherencia entre unas medidas y otras. 
Inicialmente, se presenta un diagnóstico del sistema de movilidad en Bogotá, D.C., haciendo énfasis en los planteamientos de la administración y la normativa para estructurar el sistema de transporte y desincentivar el uso del automóvil privado, para luego, como tema central, analizar la actual oferta de transporte público, específicamente en el tema de cubrimiento dentro de la ciudad $\mathrm{y}$, finalmente, concluir con la reflexión sobre el camino por seguir para ajustar la coherencia entre el escenario deseado y el que se está construyendo.

\section{Materiales y M étodos}

La investigación base incluyó variables sociales, en el análisis espacial urbano, y abordó el tema del transporte y la estructuración urbana, en una mirada que reconoce la diferencia como elemento esencial para la toma de decisiones. Tomando conceptos de diferencia y singularidad, se reconoce al otro, no sólo en las personas, sino también en los espacios urbanos, mostrando las condiciones físicas naturales y construidas de la periferia de la movilidad urbana, a diferencia de las ya reconocidas por la planeación urbana, y del transporte en el centro de la ciudad.

Se tomaron en consideración variables físicas como la topografía, la distancia y la infraestructura, pero también variables sociales como edad, estrato socio-económico y educación, entre otras, para la caracterización de la población. Además, para la evaluación de elementos, como la seguridad y la comodidad en el transporte, se realizó un sondeo con preguntas basadas en la percepción de los modos de transporte y entrevistas a personas de diferentes zonas de la ciudad y de distintos grupos de edad, sexo, nivel de estudio y ocupación.

Para el análisis de la situación actual de la movilidad, en términos de planificación, se tomó como base información secundaria de diferentes entidades distritales públicas y privadas. Desde la normativa, el Plan de Ordenamiento Territorial y los diferentes Planes Maestros en especial el de movilidad, los planes de desarrollo y las evaluaciones de las últimas administraciones en Bogotá, que permiten construir el escenario deseado y planeado de la movilidad urbana de pasajeros, en relación con la estructura de ciudad.

Así, aunque la mayor parte de la investigación esté basada en información secundaria y datos cuantitativos, y se empleen herramientas de SIG para el procesamiento de los datos, la mirada y el análisis se realizó teniendo presentes la identificación de singularidades y diferencias, tanto espaciales como sociales, con la utilización de herramientas metodológicas, entre los que se cuentan la revisión de archivos de periódicos, televisión y cortometrajes, fotografías y la aplicación de un formulario que recoge la percepción de los usuarios en cuanto los modos de transporte, entrevistas, observación 
participante e historias orales, que permitieron un acercamiento hacia la mirada de las necesidades de los usuarios del transporte público en Bogotá, D.C.

Los resultados que se presentan en este artículo se obtuvieron mediante análisis espacial, utilizando el programa ArcG is 9.2, a partir de la información cartográfica facilitada por la Secretaría Distrital de Planeación ${ }^{1}$. El mapa de estratificación de Bogotá, D.C., el perímetro urbano y las curvas de nivel son las variables principales para generar un indicador, que muestra las áreas de la ciudad con problemas de accesibilidad al Transporte Público y evidencia la falta de cubrimiento del servicio de transporte en la ciudad, en términos cuantitativos. Para la reflexión sobre el grado de aceptación del transporte público por parte de los usuarios, se realizó la lectura de los resultados arrojados por la Encuesta de Movilidad para Bogotá, D.C. del 2005, y se complementó con un sencillo cuestionario que aborda preguntas no contenidas en la encuesta.

\section{Resultados}

A partir del análisis de la encuesta de movilidad para Bogotá, D.C. del 2005 (Alcaldía Mayor de Bogotá y DANE, 2005; Alcaldía Mayor de Bogotá y STT, 2007; Alcaldía Mayor de Bogotá y STT,
2006), se puede leer fácilmente que la mayoría de personas, en la ciudad, se movilizan en transporte público. Aun así, el actual servicio en la ciudad, no cubre toda la demanda en términos numéricos, ni de satisfacción de los usuarios.

Para determinar ese conflicto de falta de cobertura del transporte público, se realizó un indicador de accesibilidad al mismo, teniendo en cuenta la distancia a las vías, pendiente del terreno, estrato socio-económico y barreras naturales representadas en las grandes zonas verdes existentes en la ciudad, reconociéndolas como los elementos mínimos que inciden en la accesibilidad al transporte, desde una mirada de toda la ciudad.

Se presenta el cubrimiento actual de Transmilenio y del transporte público, y luego el mapa de las áreas con problemas de accesibilidad a este servicio, como elemento que permite la discusión sobre el tema del transporte urbano sostenible en las ciudades y, específicamente, en Bogotá, D.C., con el fin de plantear la discusión sobre las medidas tomadas por la administración Distrital para desincentivar el uso del automóvil privado, para los viajes cotidianos en la ciudad, y acerca de la falta de cubrimiento del transporte público, que permita acoger la totalidad de usuarios que requieren el servicio.

${ }^{-}$Las coberturas fueron entregadas para la investigación por Ia SDP en el 2008. 


\section{Discusión}

\subsection{M ovilidad en Bogotá, D.C. y me- didas desde la administración para mejorarla}

Ante la preocupación mundial por la sostenibilidad ambiental, que incluye temas como el calentamiento global y la destrucción de la capa de ozono, se incluye la reflexión sobre el transporte urbano sostenible y cómo aminorar la contribución de las fuentes móviles en la contaminación (Ciudad Humana, 2006). Uno de los problemas geográficoambientales urbanos más apremiantes en la actualidad es la movilidad de personas, tanto por las múltiples formas de contaminación que suponen las alternativas de solución adoptadas, como por la segregación y desigualdad espacial que causan los diferentes grados de accesibilidad de la población y los sectores de ciudad. Es por esta razón que en varios lugares del mundo hoy se habla de transporte sostenible (Alcaldía Mayor de Bogotá y Secretaría de Tránsito y Transporte, 2006b; Alcaldía Mayor de Bogotá y Secretaría de Tránsito y Transporte, 2006c) y se buscan transportes alternativos para tratar de minimizar el impacto contaminante -al aire y el ambiente por ruido- producido por los modos de transporte motorizados, al igual que se implementan medidas para desincentivar el uso del automóvil.

En Bogotá, D.C., actualmente para las autoridades y la comunidad, es una preocupación constante y real el tema de la movilidad (Cazanoticias RCN,
2008; Radio Santafé, 2008; Semana, 2008; Duarte, 2004; Chaparro, 2002), de manera que se han implementado medidas para aminorar las emisiones al aire por fuentes móviles, que apuntan a desincentivar el uso del automóvil privado pero, también, a combatir la sobreoferta de vehículos de transporte público, sin lograr resultados duraderos, sino más bien poniendo a prueba la creatividad de los usuarios para evitarlas.

Medidas como el "pico y placa", se han convertido en la excusa para aumentar la cantidad de vehículos por hogar, entre las personas que económicamente pueden adquirirlos, o para correr las horas pico hacia periodos de tiempos anteriores o posteriores a la restricción, pero no para conformar una conciencia de la utilización de modos de transporte menos contaminantes.

Por el contrario, las medidas restrictivas, sin una satisfactoria oferta de transporte público, solamente aumentan el anhelo de las personas por adquirir su vehículo propio, como sueño para mejorar su movilidad, además del estatus social que aún significa este objeto, o la búsqueda de soluciones intermedias, como lo es actualmente la moto, que aumenta en número vertiginosamente por las calles de la ciudad y contribuye a aumentar la problemática geográfico-ambiental urbana, relacionada con la movilidad.

Desde la primera administración distrital de Antanas Mockus, entre 1995

84 S. Jiménez 
y 1997, se iniciaron campañas para desincentivar el uso del vehículo privado. La primera medida fue el "pico y placa", que inició en 1998 y restringió la movilización de automóviles durante ciertas horas del día, por números de placa de los vehículos y que, inicialmente, correspondió a la franja de 7:00 a 9:00 am y de 5:30 a 7:30 pm, de lunes a viernes, pero que cada día ha venido en aumento ${ }^{2}$, hasta hablarse hoy de establecerlo los sábados (Secretaría de Tránsito y Transporte, 2008).

Luego se propuso el "día sin carro", jornada en la cual se pretendía que no transitaran automóviles privados por las vías de la ciudad, y la totalidad de los viajes se realizaran en transporte público y modos alternativos, como la bicicleta o el caminar (Departamento Administrativo de Medio Ambiente, 2000). Aunque esta medida se ha seguido realizando año tras año, desde el 2000 , las mediciones al aire revelan que las emisiones contaminantes no se reducen considerablemente y, por el contrario, para algunos casos tienden a subir (Alcaldía Mayor de Bogotá y Departamento Administrativo de Medio Ambiente, 2007; Cider-Universidad de los Andes, 2007).

Actualmente, el "pico y placa" también funciona para los vehículos de transporte público colectivo e individual, con el mismo mecanismo que para los automóviles de uso privado, pero en el horario de 5:30 am a 9:00 pm, rotando los números de placa de lunes a sábado. Adicionalmente, desde septiembre de 2006, se implementó una medida adicional llamada "pico y placa ambiental”, que restringe el tránsito de vehículos de transporte público que no se acojan al programa de autorregulación establecido por el DAMA ${ }^{3}$, entre las 6:00 A 10:00 am, de lunes a sábado (Secretaría de Tránsito y Transporte, 2008).

Adicionalmente, se ha implementado una sobretasa al costo de la gasolina, y un aumento en el precio por hora de los estacionamientos, para motivar a los propietarios de automóviles a dejarlo en su casa y utilizar transporte público, por coste y comodidad. Pero, contrario a lo esperado, el transporte público no significa un atractivo y, generalmente, es utilizado por estricta necesidad, ante la poca posibilidad de escogencia modal de muchos bogotanos (Alcaldía Mayor de Bogotá y STT. A, 2006).

Todas estas medidas están pensadas sectorialmente y hacia uno solo de los efectos contaminantes y de los problemas de la movilidad en la ciudad: las emisiones de partículas al aire por fuentes móviles. Si bien el uso del transporte público está considerado como una de las medidas por tomar para una movilidad urbana sostenible, no se pueden olvidar objetivos básicos del sistema de transporte urbano, como lo

\footnotetext{
En la actualidad I a restricción para el vehículo privado va de 6:00 a 9:00 am y de 4:00 a 7:00 pm

Actual mente Secretaría Di strital de Medi o Ambi ente, SDMA.
} 
es la provisión del servicio a todos los habitantes, en buenas condiciones de accesibilidad. De esta manera, si se pretende acoger la mayor cantidad de viajes diarios en Bogotá, D.C., en transporte público, es necesario evaluar la oferta y el cubrimiento de este servicio.

\subsection{Movilidad en Bogotá, D.C. y el cubrimiento del transporte público}

Desde 1999, se planteó para Bogotá, D.C. la construcción y puesta en marcha del Sistema Transmilenio, basado en la tecnología de buses articulados de plataforma alta, sobre carriles exclusivos (Hidalgo, 2004; Transmilenio S.A, 2003). Inicialmente, cuando se planteó Transmilenio, se planearon diez fases de corredores troncales en prácticamente todas las vías importantes de la ciudad, para colmar la demanda total de viajes diarios en un horizonte a largo plazo (Alcaldía Mayor de Bogotá y STT, 2006c; Alcaldía Mayor de Bogotá y STT, 2004).

Actualmente, se han construido la fase I y II, y se debate sobre la conveniencia de la construcción de la fase III, sobre todo en los corredores de la carrera séptima y la calle 26 , en donde académicos y agrupaciones sociales $\mathrm{y}$ comunales han sentado su negativa al proyecto, ante los efectos espaciales que la implementación del sistema trae consigo y la poca efectividad en la solución de la movilidad en la ciudad, que estos corredores significarían.

Siendo Transmilenio la bandera del transporte público distrital, se realizó un ejercicio sencillo para determinar el área de cubrimiento, para generar áreas de influencia lineal (búfer) a lo largo de los corredores troncales construidos y las rutas alimentadoras en funcionamiento (Figura 1). Con $500 \mathrm{~m}$ a cada lado de los corredores troncales y 300 $\mathrm{m}$ en las rutas alimentadoras ${ }^{4}$, se puede observar que Transmilenio llega al $36,7 \%$ del área urbana de la ciudad ${ }^{5}$, para explicar, al menos de forma parcial, por qué sólo el $12 \%$ de viajes se realizan en este modo (Alcaldía Mayor de Bogotá y STT, 2007; Alcaldía Mayor de Bogotá y STT, 2006a).

El otro componente del transporte público en Bogotá, D.C. y el que acoge el mayor porcentaje de viajes en la ciudad, es el transporte público colectivo. Así que para complementar el ejercicio de comprobación de cubrimiento del sistema, se realizaron buffer sobre las vías por las cuales transitan buses, busetas y colectivos, tomando como referencia el área de cálculo de cubrimiento de las rutas alimentadoras $^{6}$, y luego se superpuso al realizado para Transmilenio.

\footnotetext{
Las distancias manejadas para generar los búfer son las mismas utilizadas en el cálcul o inicial y diseño de Transmilenio (Transmilenio S. A, 2005).

5 El área de cubrimiento de Transmilenio, calculada con los búfer, fue de 14.071 Ha y el área de Bogotá, D. C., dentro del perímetro urbano, es de 38.291, $40 \mathrm{Ha}$.

6 Es decir, 300 má cada I ado de I as vías (Transmilenio S. A, 2005).
} 
El ejercicio mostró que, aunque el transporte público abarca gran parte de la ciudad, 30.865,20 Ha $(80,6 \%)$, quedan zonas excluidas del servicio, o fuera de las distancias convenientes para acceder fácilmente a éste $\mathrm{y}$, por lo tanto, comunidades sin servicio de transporte público, lo cual afecta sus posibilidades de movilidad y accesibilidad a la ciudad.

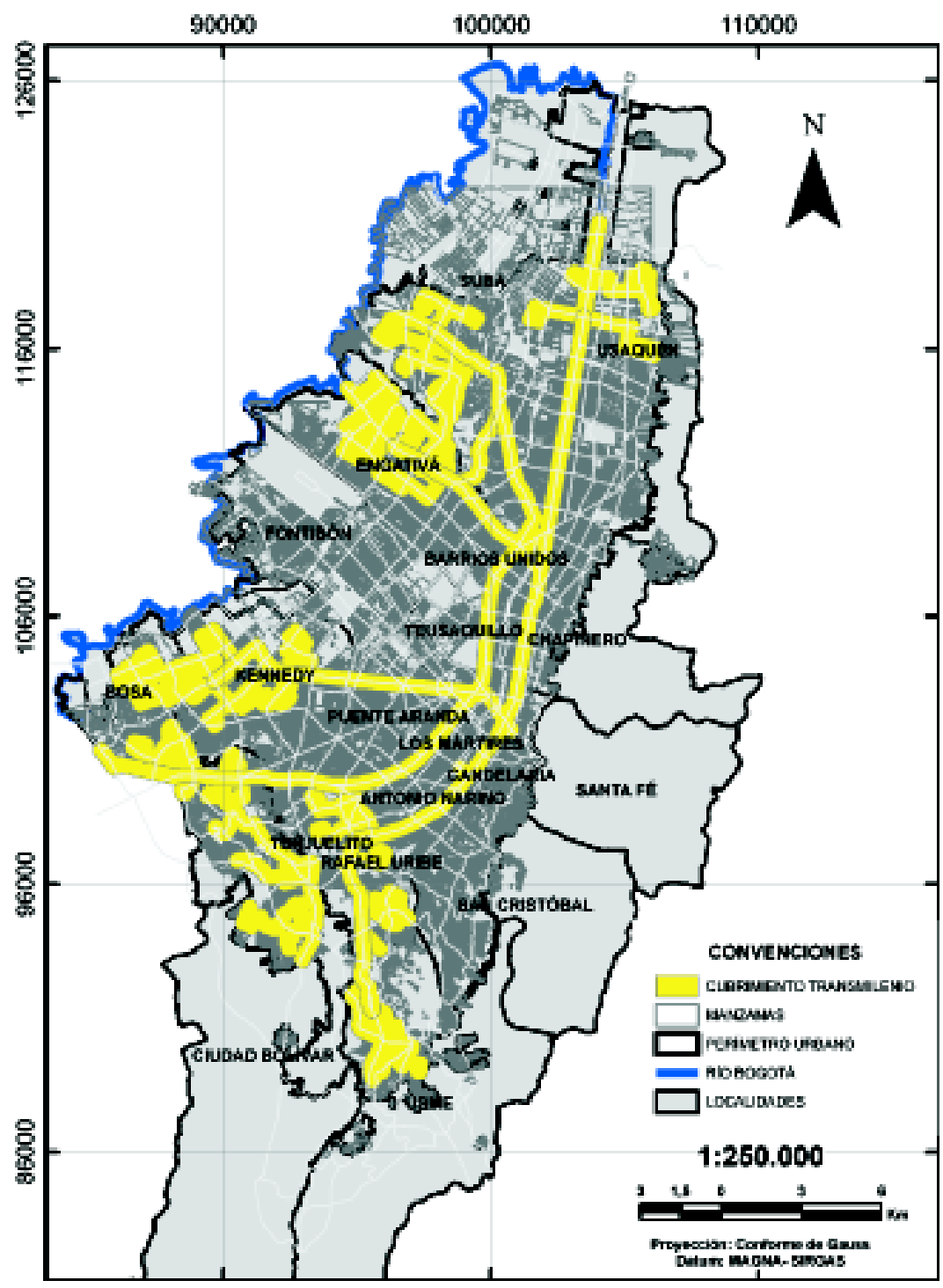

Figura 1. Áreas de cubrimiento de Transmilenio en Bogotá, D. C., 2008 Fuente: elaboración propia a partir de información entregada por la Secretaría Distrital de Planeación y Transmilenio en el 2008. 


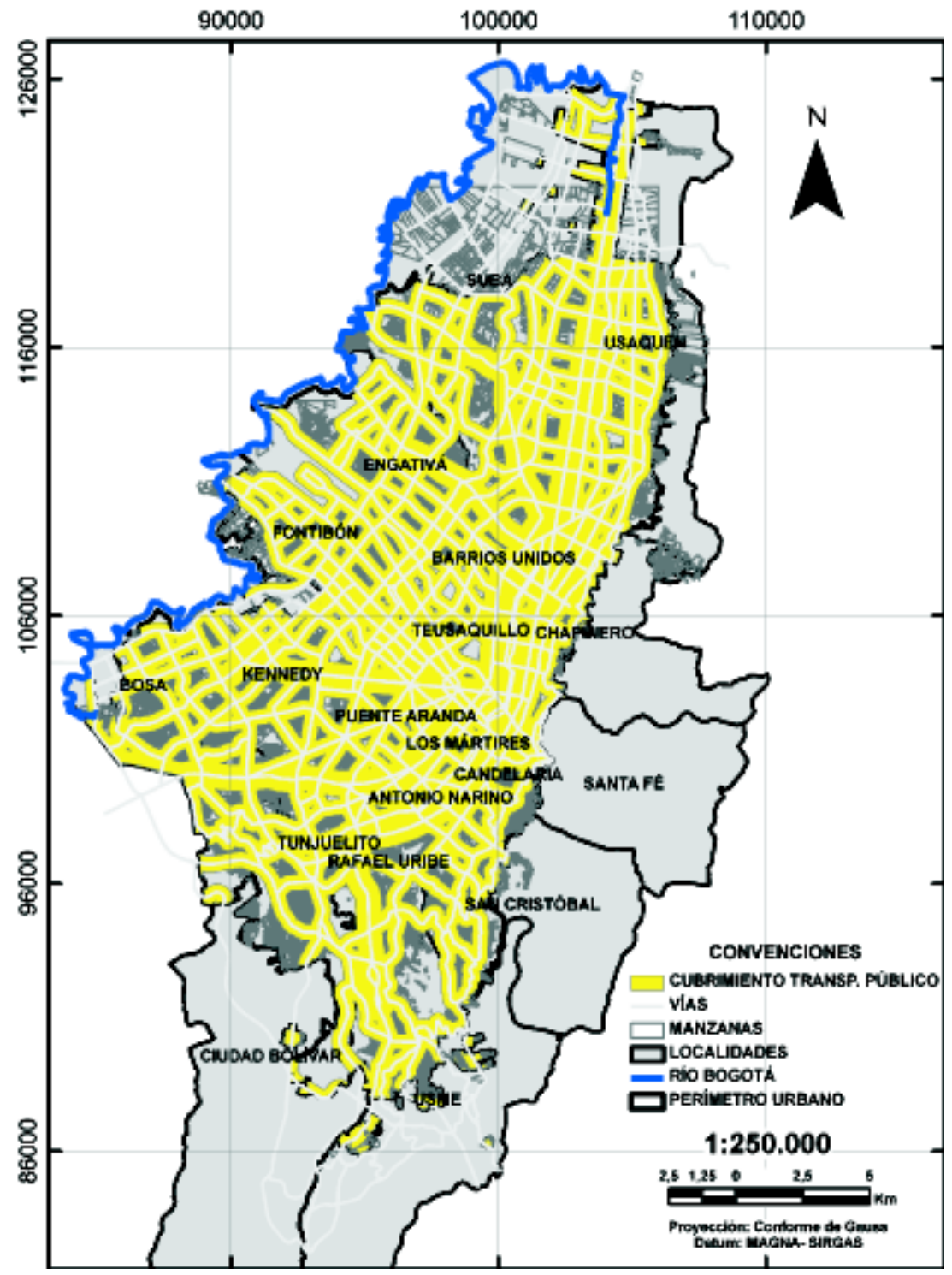

Figura 2. Área de cubrimiento del Transporte Público en Bogotá, D.C. Fuente: elaboración propia a partir de información entregada por la Secretaría Distrital de Planeación y SDM, en el 2008. 
Para hacer más exacta la evaluación del cubrimiento en área del transporte público en Bogotá, D.C., y habiendo reconocido que el espacio, además de ser medido puede ser cualificado, se realizó un análisis multivariado que incluyó las variables: pendiente del terreno, estrato socio-económico y barreras naturales representadas en las grandes zonas verdes existentes en la ciudad, reconociéndolas como los elementos mínimos que inciden en la accesibilidad al transporte, desde una mirada de toda la ciudad (Chías et al., 2001; Chías y Martínez, 2004).

Para la determinación de las áreas de la ciudad con problemas de accesibilidad al transporte público mediante el análisis multivariado, inicialmente se releyeron las distancias especificadas en los búfer de cubrimiento, ampliando el rango hasta $800 \mathrm{~m}$, clasificándolos según se muestra en la Tabla 1.

Tabla 1. Clasificación cualitativa de accesibilidad según distancias a los corredores de Transporte Público

\begin{tabular}{|c|c|c|}
\hline $\begin{array}{c}\text { R angos de distancia a la malla } \\
\text { vial arterial e intermedia }(\mathbf{m})\end{array}$ & $\begin{array}{c}\text { R angos cualitativos } \\
\text { de accesibilidad }\end{array}$ & $\begin{array}{c}\text { Calificación de 0 a 1 según } \\
\text { el nivel de accesibilidad }\end{array}$ \\
\hline 0 a 100 & Muy buena accesibilidad & 0,98 \\
100 a 300 & Buena accesibilidad & 0,75 \\
300 a 500 & Accesibilidad aceptable & 0,50 \\
500 a 800 & Accesibilidad difícil & 0,25 \\
Más de 800 & Sin accesibilidad & 0 \\
\hline
\end{tabular}

Fuente: elaboración propia a partir del análisis del diseño de Transmilenio, 2005 y Lastra, 2005. 


\section{color}

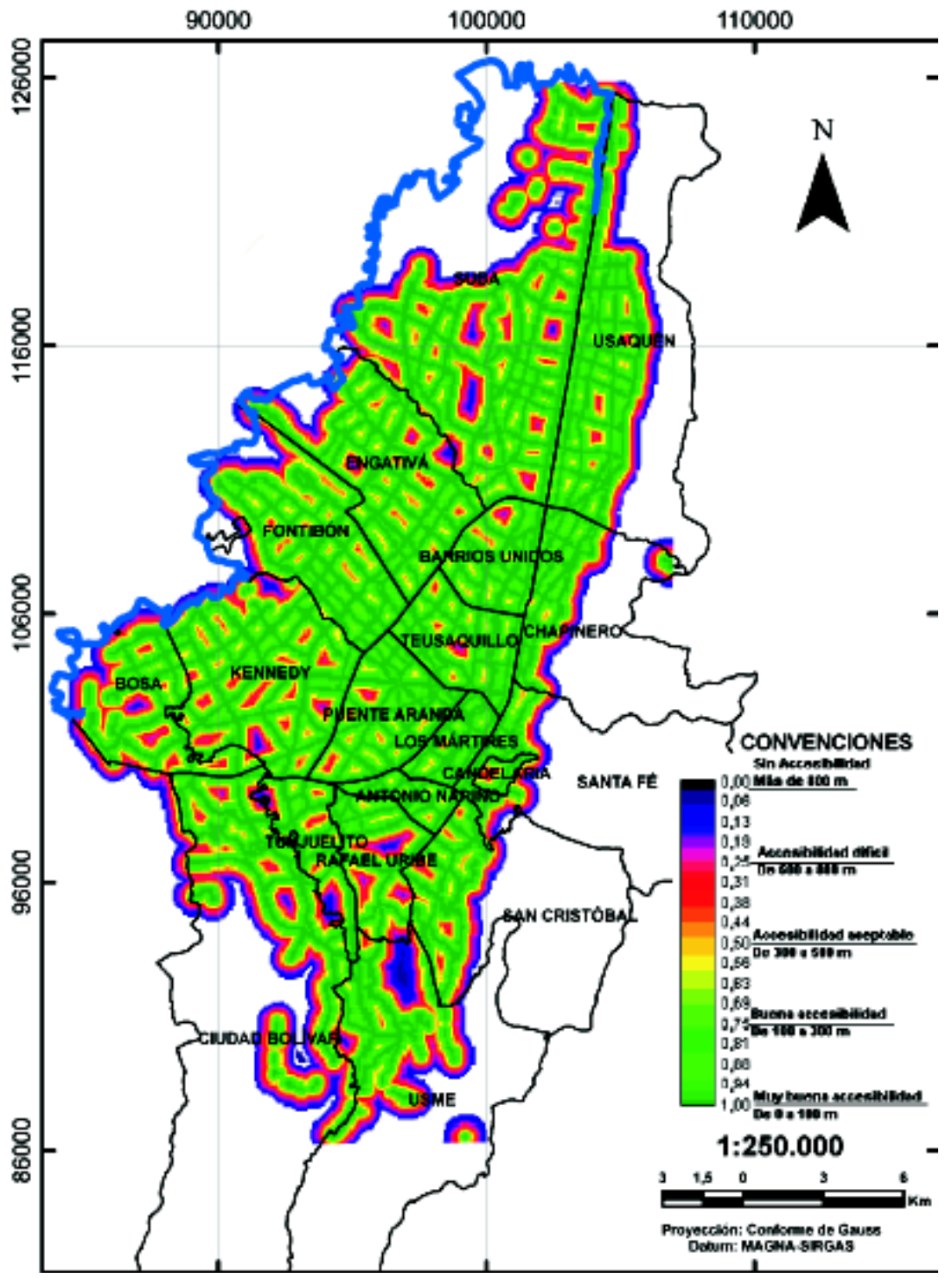

Figura 3. Accesibilidad al transporte público por rangos de distancia a las vías. Fuente: elaboración propia en Idrissi. 
A partir de las distancias establecidas en los rangos de accesibilidad, se asignó una calificación desde 0 hasta 1 , en donde 0 son los espacios a más de $800 \mathrm{~m}$ de las vías, sin accesibilidad al transporte público, y a medida que se acerca al 1 , el grado de accesibilidad aumenta, como lo muestra la Figura 3, en donde el verde evidencia las áreas con mejor accesibilidad y desde el color amarillo hasta el negro las distancias a las vías aumentan, descendiendo la facilidad de acceso al transporte público.

Este análisis presenta un resultado similar al realizado con búfer, aunque las áreas con problemas de accesibilidad se ven reducidas por el aumento de la distancia hasta $800 \mathrm{~m}$.

Ahora, entrando en la caracterización de la movilidad, en relación con las pendientes del terreno, y siguiendo a Vásquez y Martín (Chías et al., 2001), el grado de la inclinación y rugosidad de las superficies conforman una limitación para el óptimo desempeño de ciertos modos de transporte, además que dirige la forma de organizar y subdividir los predios, con la conformación de trazados urbanos diferentes.

Tabla 2. Clasificación empírica de niveles de accesibilidad según las limitaciones que impone la pendiente a los modos de transporte

\begin{tabular}{|c|c|c|}
\hline Nivel de accesibilidad & Intervalo de Pendiente & Límite para vehículos \\
\hline Sin Acceso & Mayor a $63 \%$ & Muy inclinado \\
Difícil Acceso & 12 a $63 \%$ & Límite para automóviles \\
Acceso Aceptable & 5 a $12 \%$ & Excesiva para ciclistas normales \\
Buen Acceso & Menor al $5 \%$ & Casi horizontal \\
\hline
\end{tabular}

Fuente: elaboración propia a partir de Vásquez y Martín. En: Chías, 2001. 


\section{color}

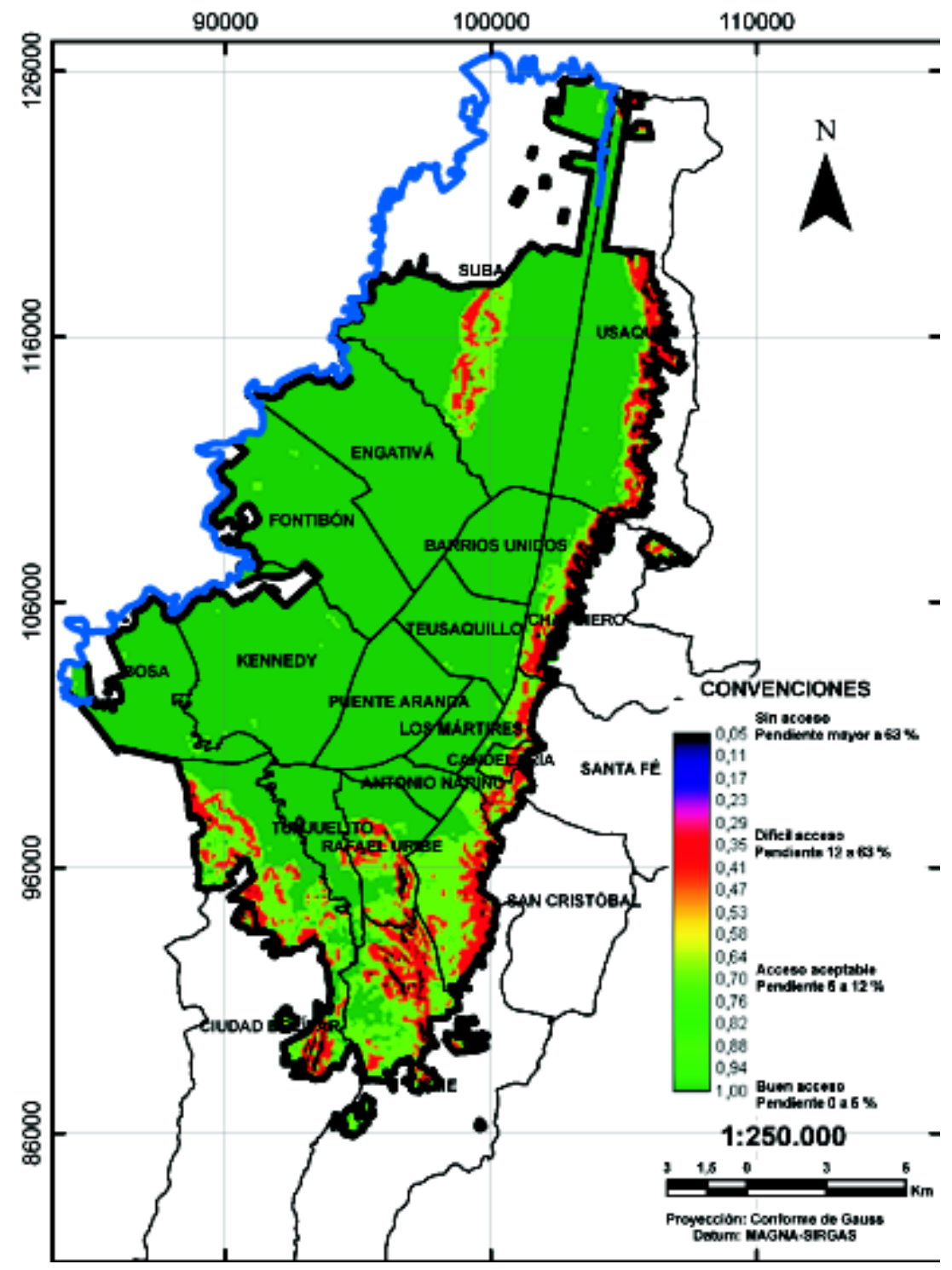

Figura 4. Accesibilidad al transporte público por intervalos de pendiente Fuente: elaboración propia en Idrissi. 
Se partió de considerar que existen unos grados de pendiente de mayor o menor accesibilidad, en relación con la cantidad de modos de transporte que pueden tener la posibilidad de atender una zona determinada. Para este análisis, la pendiente se incluyó según los niveles de accesibilidad que expone la Tabla 2, teniendo presentes las limitaciones que esta característica espacial impone a los modos de transporte, y conociendo que el servicio público basa su parque automotor en vehículos convencionales -Articulados, buses, busetas y colectivos-, para los cuales cierto grado de inclinación en el terreno ya es una condicionante para su implementación. Así, se calificó de 0 a 1 , como se explicó con la variable distancia.

La Figura 4 muestra en verde las áreas con buena accesibilidad dentro del perímetro urbano de Bogotá, D.C. y en rojo las zonas que, por su elevada pendiente, se consideraron sin acceso al transporte público de la ciudad.
Básicamente, las zonas con dificultades de acceso al transporte público por pendiente del terreno corresponden a los cerros orientales, sur-orientales y los cerros de Suba, pero hay que considerar que, en los últimos años, estas zonas de ladera han sido urbanizadas,

sobre todo hacia el sur-oriente de la ciudad, en la Localidad de Usme y Ciudad Bolívar, mediante desarrollos informales, y en el norte de Usaquén, en barrios como Lijacá, Codito y Santa Catalina, aunque también hacia Chapinero alto, en desarrollos que podrían llamarse formales, con residencias suntuosas.

La posibilidad de acceso económico al Transporte se tuvo en cuenta por la facilidad o dificultad de pago con el que cuentan los habitantes de Bogotá, D.C., según su nivel de ingresos, tomando la variable estrato socio-económico que, aunque en algunos casos no refleja literalmente los ingresos de una persona, es la información existente, a escala de Bogotá, D.C., que permite un acercamiento en este tema.

Tabla 3. Clasificación cualitativa de accesibilidad al transporte público según la capacidad económica de pago.

\begin{tabular}{|c|c|c|}
\hline $\begin{array}{c}\text { Estrato } \\
\text { socioeconómico }\end{array}$ & $\begin{array}{c}\text { Rangos cualitativos } \\
\text { de accesibilidad }\end{array}$ & $\begin{array}{c}\text { Calificación 0 a 1 } \\
\text { según nivel accesibilidad }\end{array}$ \\
\hline 5 y 6 & Muy buena & 0,98 \\
4 & Buena & 0,75 \\
3 & Aceptable & 0,50 \\
2 & Regular & 0,25 \\
1 & Mala & 0 \\
\hline
\end{tabular}

Fuente: elaboración propia. 


\section{color}

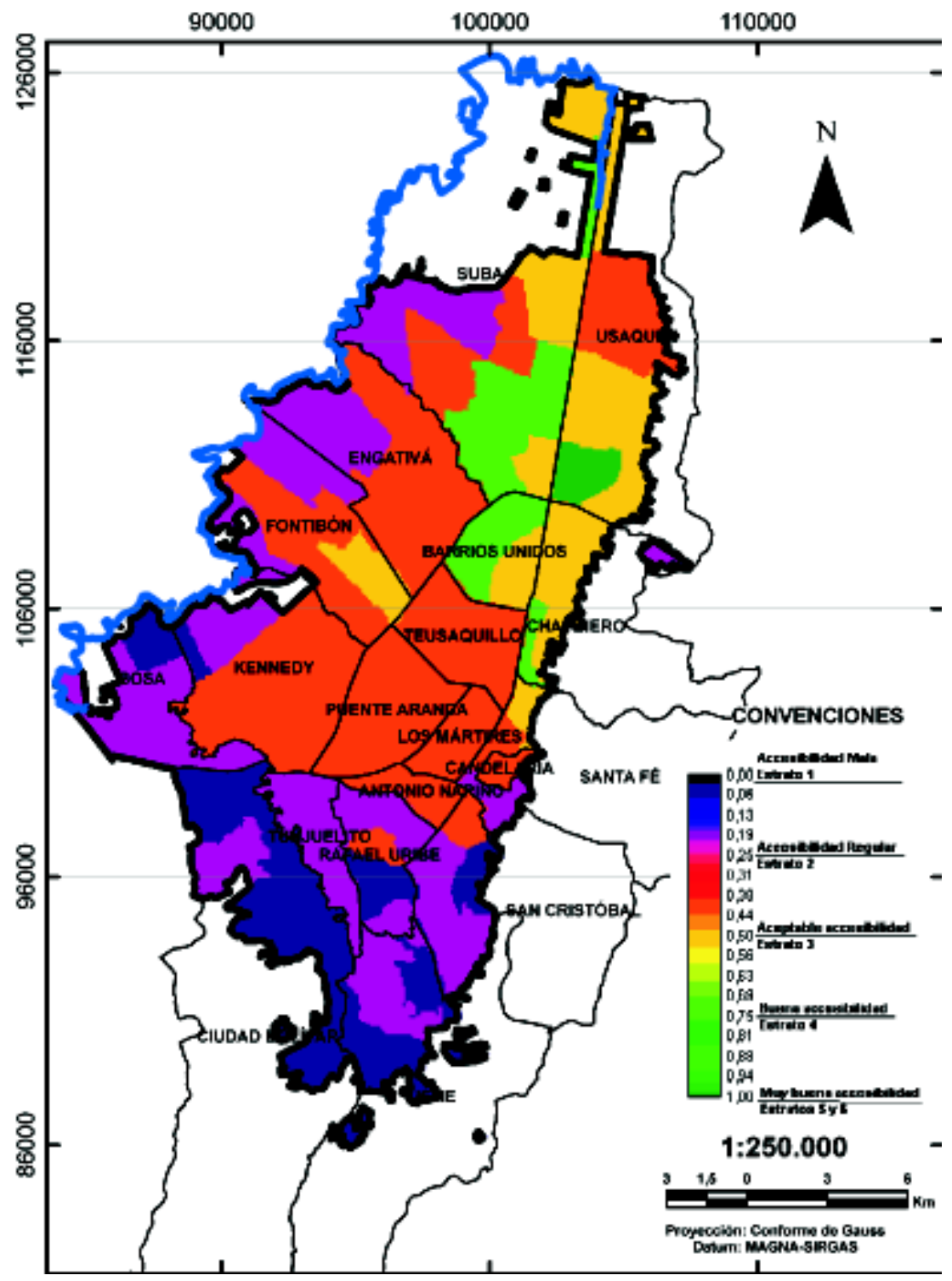

Figura 5. Accesibilidad al transporte público por posibilidad económica

Fuente: elaboración propia a partir del análisis del diseño de Transmilenio y Lastra, 2005. 
Estas tres variables expuestas se calificaron por igual, con un peso de 0.33 cada una, sin determinar una mayor importancia para alguna o variables dependientes.
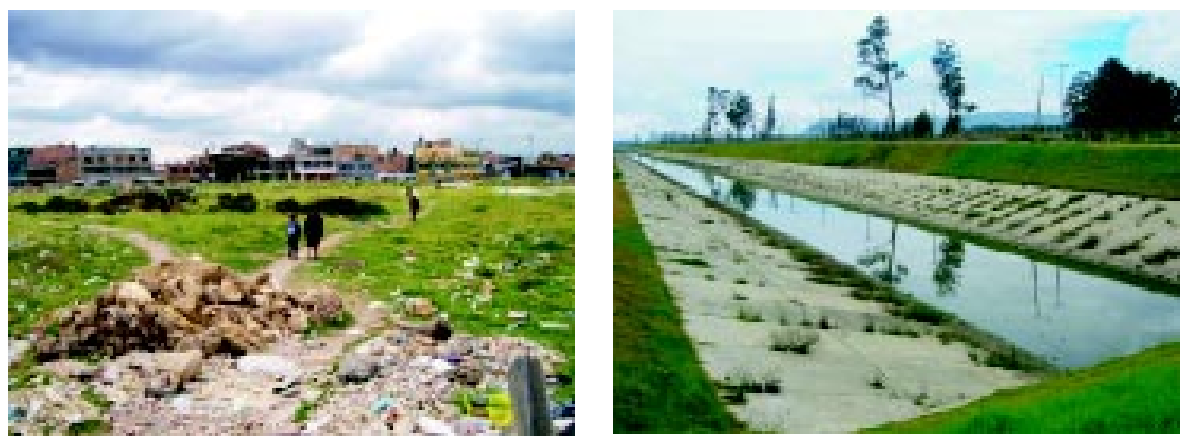

Figura 6. Espacio verde (izquierda) y canal (derecha), como barreras a la movilidad urbana

Fuente: archivo personal.

Las zonas verdes se incluyeron como limitantes para las distancias, porque, aunque necesarias en la ciudad, en grandes extensiones se convierten en barreras físicas para el desplazamiento de los habitantes y, al tener que rodearlas, representan mayores distancias, lo cual cambia el cálculo de las áreas de cubrimiento del Transporte Público.

Al realizar el análisis, combinando las variables distancia a las vías y pendientes, con la restricción de las zonas libres, se muestra en gris (1) las áreas con problemas de accesibilidad al transporte público (Figura 7). Al considerar además el estrato económico (Figura 8), se puede observar que, en este último, desaparecen varias zonas con problemas de accesibilidad en Chapinero, Usaquén y el oriente de Suba, que sí muestra la Figura 7. 


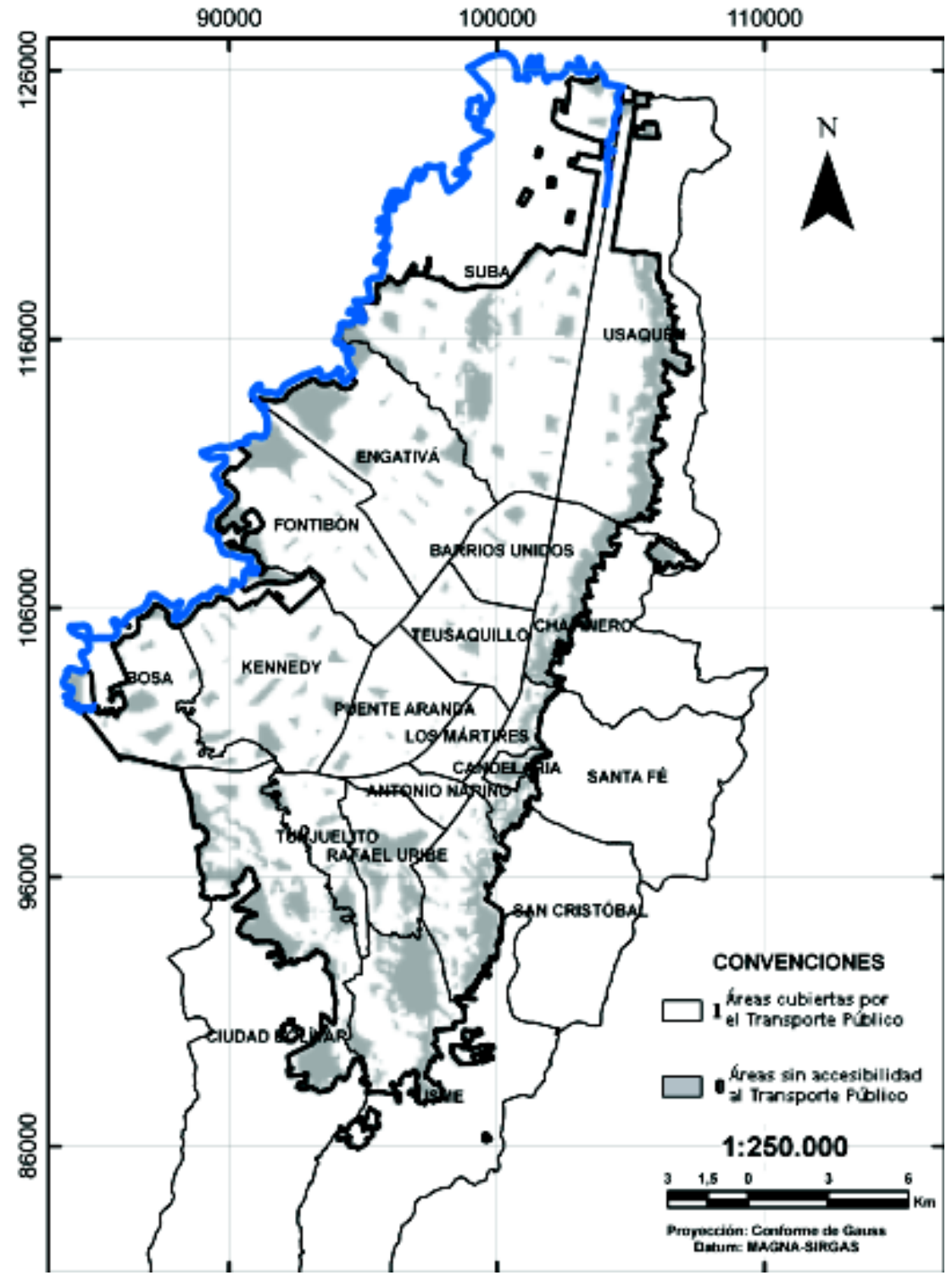

Figura 7. Áreas con problemas de accesibilidad al transporte público, considerando distancia y pendiente.

Fuente: elaboración propia. 


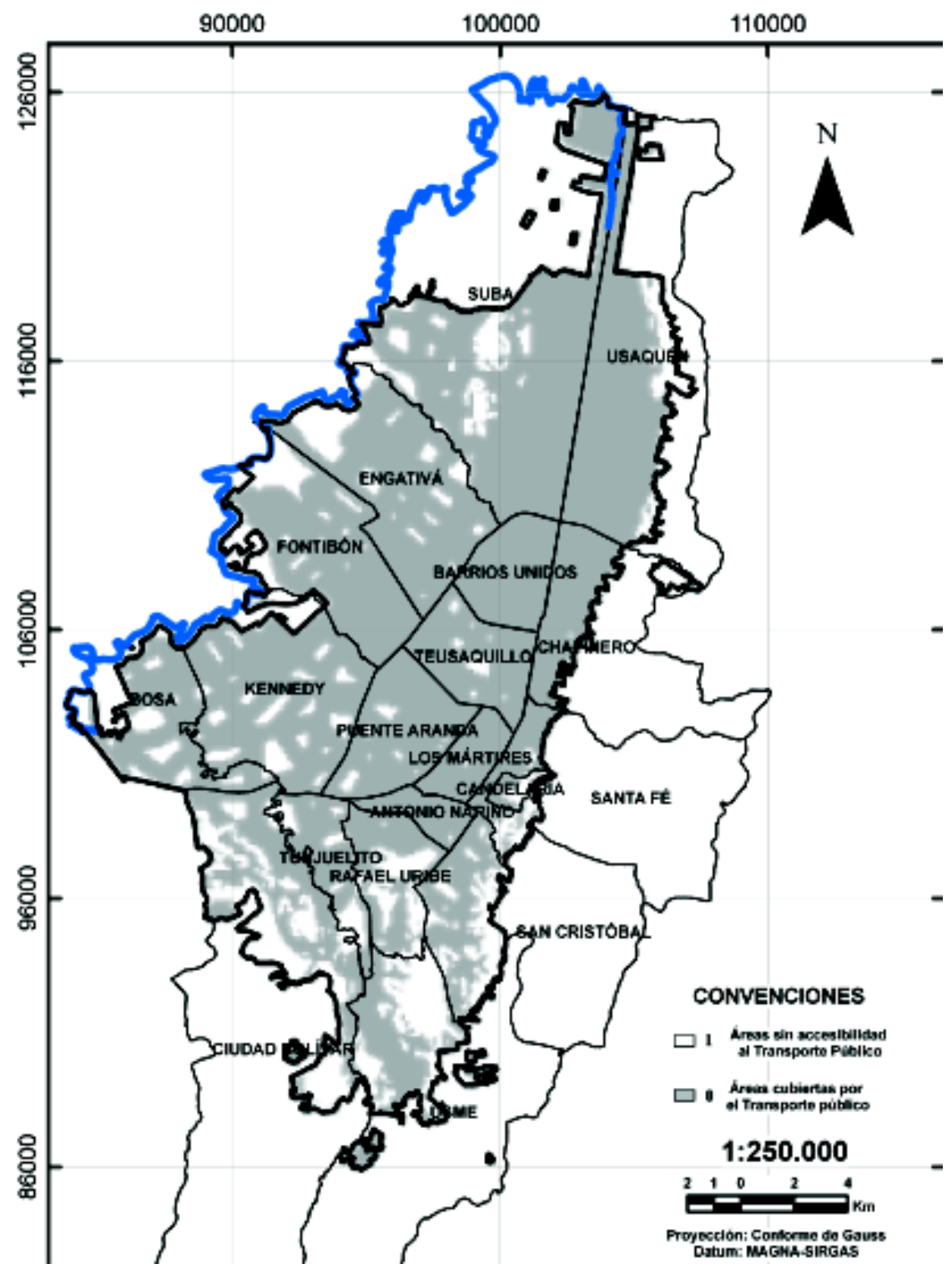

Figura 8. Áreas cubiertas por el transporte público, considerando distancias, pendientes y estrato.

Fuente: elaboración propia. 
Los estratos 5 y 6 tienen mayor posibilidad de acceder al vehículo privado o el taxi (Alcaldía Mayor de Bogotá y STT, 2007; Alcaldía Mayor de Bogotá y STT, 2006a) o, incluso, de abordar más de un transporte por desplazamiento, a diferencia de las personas que, por oferta y posibilidad de pago, dependen básicamente del transporte público y/o del Transmilenio, por lo cual se eliminan del análisis anterior (Figura 8) y no porque necesariamente tengan mejores condiciones espaciales de accesibilidad ${ }^{7}$.

Finalmente, el inverso de la Figura 8 (Figura 9), evidencia las áreas en Bogotá, D.C. con problemas de accesibilidad al transporte público ${ }^{8}$. Teniendo en cuenta que con el análisis multicriterio, la posibilidad económica de acceso al transporte redujo las áreas con problemas de accesibilidad, ubicadas en Usaquén, Chapinero y el Oriente de Suba, pero que, además, la dificultad de acceso, por las pendientes elevadas y las barreras naturales, aumentaron las áreas al nor-oriente en Usaquén y al sur y suroriente en Usme y Ciudad Bolívar, el área sin cubrimiento del transporte público suma $6.120 \mathrm{Ha}$, equivalente a un $16 \%$ del área urbana de Bogotá, D.C. Dato que, aunque no especifica la cantidad de población afectada, muestra de manera inicial un problema de cubrimiento, que deja sectores con serios problemas para el acceso al transporte público $\mathrm{y}$, por ende, a los servicios urbanos de la ciudad.

Así mismo, es difícil esperar que las medidas para desincentivar el uso del vehículo privado arrojen resultados positivos, si no existe una completa cubertura y excelente servicio desde el transporte público, que pueda cubrir la demanda de viajes en la ciudad.

Si actualmente, con la oferta existente en transporte público y privado, legal e ilegal que ofrece la ciudad, hay áreas y comunidades en Bogotá, D.C. con problemas de accesibilidad y movilidad, es contradictorio buscar atraer más personas a un sistema aún ineficiente, que omite las diferencias espaciales y socioeconómicas de la población, al igual que sus necesidades y requerimientos en cuanto su movilidad.

Se reconocen los problemas de muilidad y accesibilidad en sectores de estrato 5 y 6 , principales usuarios del vehículo privado, pero también se considera que obedecen a otras variables, como el trazado urbano y configuración de los barrios, que requi ere un estudio específico, el cual, además, busque la forma de desestimular el uso del automóvil.

8 Estos polígonos resultantes fueron depurados, teniendo en cuenta, como míni mo, un área de 1 Ha que, aproxi madamente, corresponde a una manzana. Además, se exami nó y comparó con el plano de manzaneo, para confirmar que estuvieran en zonas habitadas. 


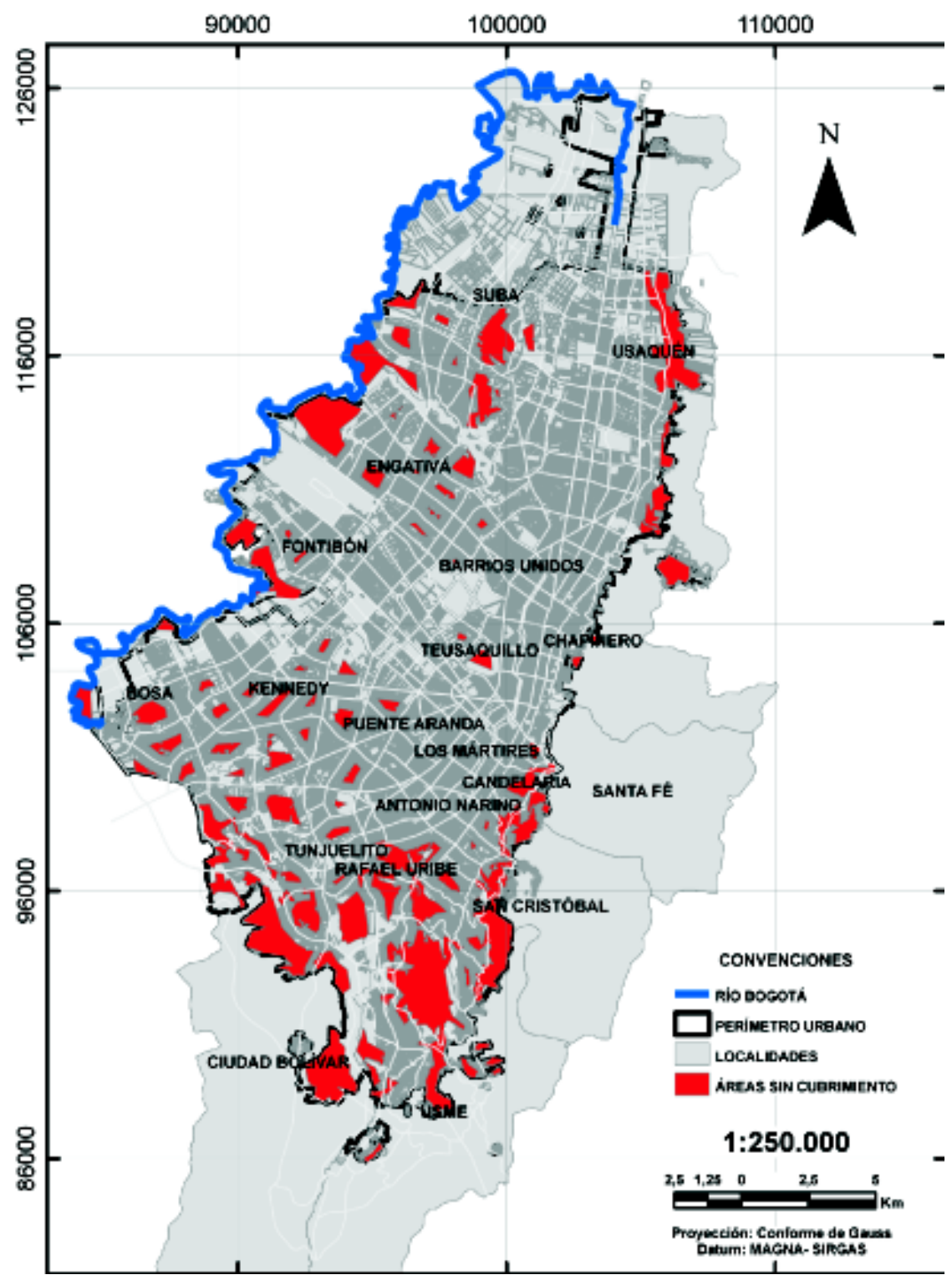

Figura 9. Áreas de Bogotá, D.C. con problemas de accesibilidad al Transporte Público Fuente: elaboración propia a partir de información entregada por la Secretaría Distrital de Planeación y SDM en el 2008 


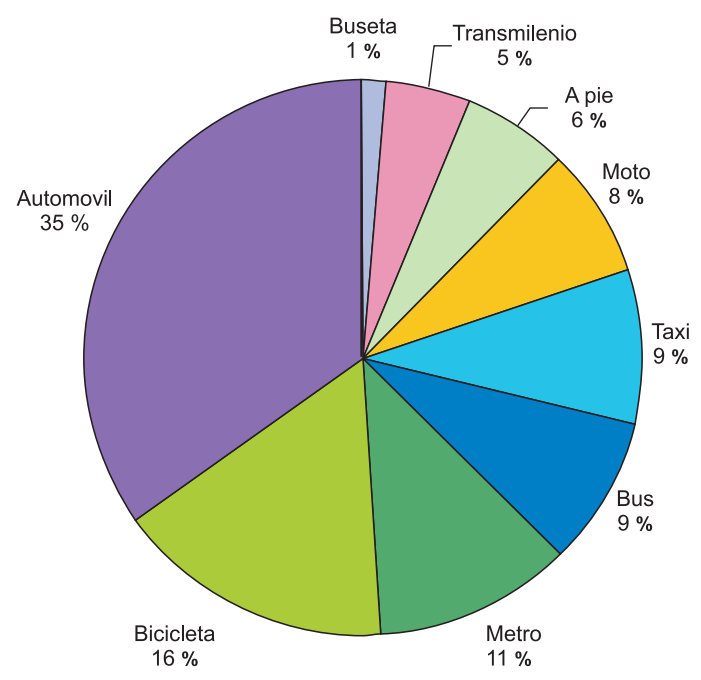

Figura 10. Modo de transporte en el que le gustaría viajar diariamente Fuente: elaboración propia

Por otra parte, en cuanto al grado de satisfacción por el servicio de transporte público en Bogotá, D.C., al preguntar a la gente en qué modo de transporte le gustaría viajar, la preferencia por el automóvil se evidencia con el $35 \%$ de los encuestados (Figura 10), aunque sólo el $6 \%$ de ellos viajen diariamente en este modo, lo cual proporciona una idea de la proyección de muchos bogotanos por adquirir vehículo, a pesar de las restricciones adelantadas por el Distrito para su movilización y de las ideas de la movilidad sostenible.

Al comprobar la disposición para usar uno u otro modo de transporte urbano (Figura 11), se observa que el automóvil privado todavía sigue arraigado en las preferencias de las personas, ya que el $80 \%$ de los entrevistados señalaron que estarían dispuestos a usarlo y el 50\% de estos lo colocaron como el modo que más dispuestos estarían a utilizar. 


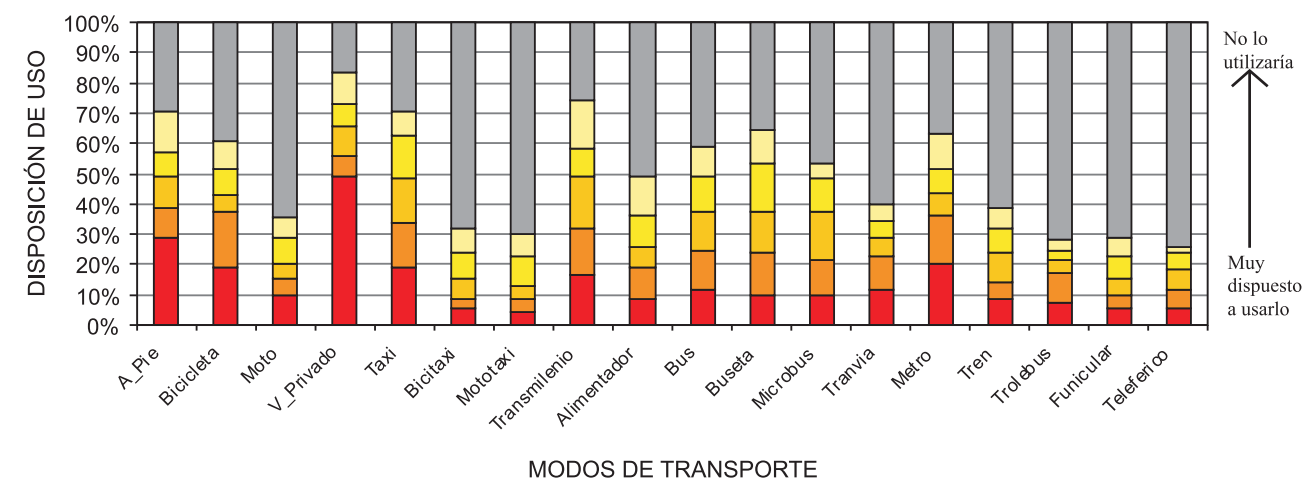

Definitivamente no lo utilizaría

Figura 11. Disposición para utilizar modos de transporte urbanos Fuente: elaboración propia

Sin embargo, los modos de transporte masivo y público obtuvieron una buena calificación, dejando notar que es posible acoger y mantener, como usuarios de estos modos, a muchos bogotanos, si el servicio y el cubrimiento mejora, lo cual lograría un mejor funcionamiento en la ciudad, en consideración de los tiempos de llegada y salida de los habitantes en las diferentes zonas, y las distancias que tienen que recorrer antes de poder abordar un alimentador o un bus urbano.
Al igual, las quejas sobre incomodidad, coste económico y tiempos de espera en Transmilenio, y tiempos de viaje en el transporte público colectivo, están a la orden del día entre los usuarios. Es necesario, desde la administración, revaluar su política de transporte en la ciudad y fortalecer el transporte público, antes de pedir a los ciudadanos bajarse de su carro o del sueño de tenerlo, para colaborar con una movilidad sostenible utópica, sectorizada y entendida sólo como contaminación al aire por emisión de partículas. 


\section{Literatura citada}

Alcaldía Mayor de Bogotá. 2001. Para transporte público colectivo, Pico y Placa Ambiental comienza a regir el primero de septiembre. http://www.bogota.gov.co histo.php?idh $=16770 \&$ patron $=1 \#$. Se tuvo acceso en diciembre de 2008 .

Alcaldía Mayor de Bogotá. 2003. Decreto 469 de 2003: "Por el cual se revisa el Plan de Ordenamiento Territorial de Bogotá, D.C”. Registro Distrital 3013 del 23 de diciembre de 2003. http://www.alcaldiabogota.gov.co/sisjur/normas/Norma1.jsp?i=10998. Se tuvo acceso en noviembre de 2005.

Alcaldía Mayor de Bogotá 2004. Compilado del Plan de Ordenamiento Territorial. Decreto 190 de 2004: Por medio del cual se compilan las disposiciones contenidas en los Decretos. Distritales 619 de 2000 y 469 de 2003". Bogotá, D.C. Registro Distrital 3122 del 22 de junio de 2004. http://www.alcaldiabogota.gov.co/sisjur/normas/Norma1.jsp?i=13935. Se tuvo acceso en noviembre de 2005.

Alcaldía Mayor de Bogotá. 2006. Decreto 319 de 2006 del Alcalde Mayor. Por el cual se adopta el Plan Maestro de Movilidad para Bogotá Distrito Capital, que incluye el ordenamiento de estacionamientos. Registro Distrital 3596. Agosto 18 de 2006. http:/ /www.alcaldiabogota.gov.co/sisjur/normás/Norma1.jsp?i=21066. Se tuvo acceso en diciembre de 2006

Alcaldía Mayor de Bogotá y Secretaría de Tránsito y Transporte, STT. 2001. POT: Resumen Plan de Ordenamiento Territorial. Construir ciudad. Bogotá, D.C.: Panamericana Formas e Impresos.

Alcaldía Mayor de Bogotá y Secretaría de Tránsito y Transporte, STT. 2004. Movilidad en el ordenamiento territorial. Presentada en el Foro: El transporte masivo de Bogotá D.C. Reflexiones para el futuro.

Alcaldía Mayor de Bogotá y Secretaría de Tránsito y Transporte, STT. 2005a. Plan Maestro de Movilidad. Bogotá, D.C.: Panamericana Formas e Impresos. Departamento Administrativo de Planeación Distrital.

Alcaldía Mayor de Bogotá y Secretaría de Tránsito y Transporte, STT. 2005b. Plan Piloto de Movilidad del Centro. Bogotá, D.C.: Panamericana Formas e Impresos.

Alcaldía Mayor de Bogotá y Secretaría de Tránsito y Transporte, STT. 2006a . Encuesta de Movilidad. Presentación de socialización. Bogotá Solidaria, una ciudad sin diferencias.

Alcaldía Mayor de Bogotá y Secretaría de Tránsito y Transporte, STT. 2006b. Formulación del Plan Maestro de Movilidad para Bogotá D.C. Presentación de socialización.

Alcaldía Mayor de Bogotá y Secretaría de Tránsito y Transporte, STT. 2006c. Formulación del Plan Maestro de Movilidad para Bogotá D.C. que incluye ordenamiento de estacionamientos. Documento técnico de soporte del PMM. Informe final ajustado. 
Alcaldía Mayor de Bogotá y Secretaría de Tránsito y Transporte, STT. 2007. Encuesta de Movilidad. Bases, metodología y diccionario de datos. Última versión entregada en junio. Bogotá, D.C.

Alcaldía Mayor de Bogotá y Departamento Administrativo de Estadística, DANE. 2005. Encuesta de Movilidad Urbana Bogotá 2005. Presentación de ocialización. Bogotá, D.C.

Alcaldía Mayor de Bogotá y Departamento Administrativo de Medio Ambiente. 2007. Jornada "Sin mi carro en Bogotá". Consolidado. Red de Calidad del aire. Bogotá, D.C.

Cazanoticias, RCN Televisión. 2008. Denuncia sobre problemas de accesibilidad al transporte público en Cazucá, barrio al sur de Bogotá, en límites con Soacha. Bogotá, D.C.: 24 de marzo, emisión de las 7:00 pm.

Chaparro, I. 2002. Evaluación del impacto socioeconómico del transporte urbano en la ciudad de Bogotá. El caso del sistema de transporte masivo, Transmilenio. Serie Recursos Naturales e Infraestructura No. 48. CEPAL. División de Recursos Naturales e Infraestructura, Unidad de Transporte. Publicación de las Naciones Unidas. Santiago de Chile. 77 p.

Chías, L.; Iturbe, A. y Reyna, F. 2001. Accesibilidad de las localidades del Estado de México a la red carretera pavimentada: un enfoque metodológico. Investigaciones Geográficas, 46:116-130.

Chías, L. y Martínez, A. 2004. La inseguridad vial en México: Paradigmas para su análisis geográfico. Perspectiva Geográfica 10: 145-163.

CIDER-Universidad de los Andes. 2007. Debate sobre Movilidad en Bogotá D.C. “Análisis sobre el día sin carro y sus efectos sobre la Movilidad el Modelo de Ciudad". En: Seminario de Maestría: "Desarrollo en Bogotá D.C.: Instituciones, Actores, y política pública”.

Ciudad Humana. 2006. Towards Carfree Cities Conference VI. Bogotá, D.C.

Departamento Administrativo de Medio Ambiente. 2000. El primer día sin carro, febrero de 2000. http://observatorio.dama.gov.co/index.php?n1 $=2 \& n 2=23 \& n 3=237$ $\&$ contenido $=81$. Se tuvo acceso en diciembre de 2008 .

Duarte, E. 2004. Transporte urbano sostenible en Bogotá. Duarte Guterman y CIA. Presentada en el Foro: El transporte masivo de Bogotá D.C. Reflexiones para el futuro.

El Espectador. 2007. Contaminación atmosférica debilitó centro histórico. La mala calidad de los combustibles en especial el que usan los vehículos de transporte, debilitan las estructuras en el centro de la ciudad y afectan además, la salud de habitantes y peatones. Bogotá, D.C.: martes 18 de septiembre. http://www.elespectador.com/impreso/ Secciones/Detalles.aspx?idNoticia $=15340$. Se tuvo acceso en septiembre de 2007. 
El Tiempo. 2006a. Rajan Plan Maestro de Movilidad. Bogotá, D.C.: 22 de marzo. http:// www.eltiempo.com/archivo/documento/MAM-1962974. Se tuvo acceso den Septiembre de 2008

El Tiempo. 2006b. La mala movilidad le cuesta muy caro a la ciudad. Bogotá, D.C.: 31 de julio. http://www.eltiempo.com/archivo/documento/CMS-3042234. Se tuvo acceso den Septiembre de 2008

El Tiempo. 2008a. Controlar el tránsito en Bogotá, un problema que se salió de las manos. Bogotá, D.C.: 19 de enero. http://www.motor.com.co/noticias_precios /vehiculos./ 19deenerode2008/ARTICULO-WEB NOTA INTERIOR MOTOR V2-3756213.html. Se tuvo acceso en enero de 2008

El Tiempo. 2008b. No habrá Sistema Integrado antes del 2010, anunció el Secretario de Movilidad del Distrito. Bogotá, D.C.: 19 de mayo. http://www.eltiempo.com/archivo/ documento/CMS-4173832. Se tuvo acceso en mayo de 2008

Hidalgo, D. 2004. Tecnologías de Transporte Masivo. Presentada en el Foro: El transporte masivo de Bogotá D.C. Reflexiones para el futuro.

Lastra, I. de La. 2005. La accesibilidad en la ordenación territorial de las aglomeraciones urbanas en Andalucía. Sevilla: Universidad de Sevilla.

Radio Santafé. 2008. Medidas para evitar trancones en Bogotá. 13 de marzo. http:// www.radiosantafe.com/2008/03/13/medidas-para-evitar-trancones-en-bogota/. Se tuvo acceso en septiembre de 2008

Secretaría de Tránsito y Transporte. 2008. Particular, Público, Normas y excepciones. http:/ www.transitobogota.gov.cocontenido.asp?plantilla $=7 \&$ pub_id $=93 \&$ pag_id $=286 \&$ cat_id $=87$. Se tuvo acceso en diciembre de 2008.

Semana. 2008. ¿Fin a la guerra del centavo? En seis meses la administración Moreno debe poner a andar el remedio para ponerle fin al caótico transporte público de la ciudad. ¿Podrá lograrlo? En: Revista Semana, Bogotá, D.C.: 17 de mayo de 2008. http:// www.semana.com/wf_InfoArticulo.aspx?IdArt $=111913$. Se tuvo acceso en septiembre de 2008

Transmilenio S.A. y Alcaldía Mayor de Bogotá. 2003. Transmilenio: La Joya de Bogotá. Bogotá, D.C.: Panamericana Formas e Impresos S.A.

Transmilenio S. A.. 2005. Cinco años construyendo futuro. Transmilenio S.A. Bogotá, D.C.: Panamericana Formas e Impresos S.A.

Fecha de recepción: 16 de diciembre de 2008

Fecha de aprobación: 29 de diciembre de 2008 